ISSN1027-5495. Functional Materials, 23, No.3 (2016), p. 463-467

doi:http://dx.doi.org/10.15407/fm23.03.463

(C) 2016 - STC “Institute for Single Crystals"

\title{
Target prediction in blast furnace based on BP network optimized by variable neighborhood PSO
}

\author{
Yang Kai, ${ }^{1,2}$ Zhijun $\mathrm{He}^{1}$ \\ ${ }^{1}$ School of materials and metallurgy, University of Science and Technology \\ LiaoNing, \\ LiaoNing 114051, China, \\ ${ }^{2}$ School of Software, University of Science and Technology LiaoNing, \\ LiaoNing 114051, China
}

Received June 22,2016.

\begin{abstract}
This paper combine the improved PSO algorithm (Analysis of Particle Swarm Optimization Algorithm) with the BP neural network for prediction of Silicon content in hot metal. Firstly, the varying visual mechanism is drawing into the standard PSO through changing the neighbor structure dynamically with each particles, in order to enhance the local and global searching ability in particle swarm. Afterwards, the improved algorithm is used to optimize the weights and threshold of BP neural network to avoid falling into local extremum. Finally, the prediction model of Si content in hot metal is built based on BP network optimized by Variable neighborhood PSO. The average relative error of the prediction model is $6.7 \%$ based on the data from blast furnace.
\end{abstract}

Keywords: particle swarm optimization, neural network, silicon content, prediction.

Предлагается улучшенный алгоритм PSO для прогнозирования содержания кремния в горячем металле. Средняя относительная погрешность модели прогнозирования составляет $6,7 \%$ на основе данных из доменной печи.

Прогнозування змісту кремнію в гарячому металі за допомогою алгоритму PSO. Ян Кай, Шун Xi

Пропонуеться покращений алгоритм PSO для прогнозування вмісту кремнію в гарячому металі. Середня відносна похибка моделі прогнозування становить $6,7 \%$ на основі даних 3 доменної печі.

\section{Introduction}

The silicon content in blast furnace hot metal is an important index to measure the quality of pig iron and metallurgical technology. The change of silicon content directly reflects the stability of the process[1,2]. Therefore, realizing the silicon content in hot metal and it's varying tendency in real time, and making accurate forecasts, have significances to conduct operating for temperature of blast furnace, reduce focal ratio and the cost of pig iron, reduce fluctuation of furnace condition, and ultimately achieve energy saving and reducing consumption.

Because the traditional prediction model based on the production mechanism of hot metal has certain limitations. Recently, Many scholars use artificial intelligence methods to predict the quality of hot metal [3-6]. This paper combine the improved PSO algorithm with the BP neural network for prediction of Silicon content in hot metal. The concept that each particles has certain visual field is firstly proposed in this paper. All the particles in the visual field of a given particle form a neighbor- 
hood structure of the particle. The visual field of each particle is changing along with iteration, leading to the varying neighborhood of particles, in order to improve the ability of the particle swarm to find the optimal solution. On this basis, the improved algorithm is used to optimize the weights and thresholds in BP neural network to avoid falling into local extremum. Later the prediction model based on BP network optimized by Variable neighborhood PSO is to be trained and validated by real data in a steel company.

\section{PSO algorithm and it's improvement}

\subsection{The standard PSO}

A group of $\mathrm{n}$ particles is flying at a certain speed in D-dimensional searching space. Each particle is a potential solution for the optimization problem to be solved. Speed determines their flight distance and direction. All the particles have a fitness value that is determined by the function of the optimization. Particles update their position and speed by following two extreme values in the process of flight, which separately represent their own flight experience and the swarm flight experience. One extreme value is the optimal solution that the particle itself has found so far, called the individual extreme value, denoted by pbest. The other extreme value is the optimal solution that the whole population has found so far, called the global extreme value, denoted by gbest. The update formula for particle position and velocity is:

$$
\begin{gathered}
v_{i d}^{k+1}=w v_{i d}^{k}+c_{1} r_{1}\left(\text { pbest }_{i d}-x_{i d}^{k}\right)+ \\
c_{2} r_{2}\left(\text { gbest }_{g d}-x_{i d}^{k}\right) \\
x_{i d}^{k+1}=x_{i d}^{k}+v_{i d}^{k+1}
\end{gathered}
$$

Where $i=1,2, \cdots, n, n$ is the size of the swarm; $d=1,2, \ldots, D, D$ is the number of dimensions, $k$ is the number of iterations; $c_{1}, c_{2}$ are two positive constants, called cognitive and social parameter respectively; $r_{1}, r_{2}$ are two random functions in the range $[0,1]$. W is called inertia weight, used to coordinate the global and local optimization ability of PSO algorithm. W usually decreases linearly with the increase of the number of iterations. In this paper, the updating formula of the inertia weight is:

$$
\begin{aligned}
& w= \\
& =\left(w_{\max }-w_{\min }\right) \times \frac{\text { maxiter }- \text { curiter }}{\text { maxiter }}+w_{\text {min }}
\end{aligned}
$$

where $w_{\max }, w_{\min }$ are the maximum and minimum values respectively of the inertia weight

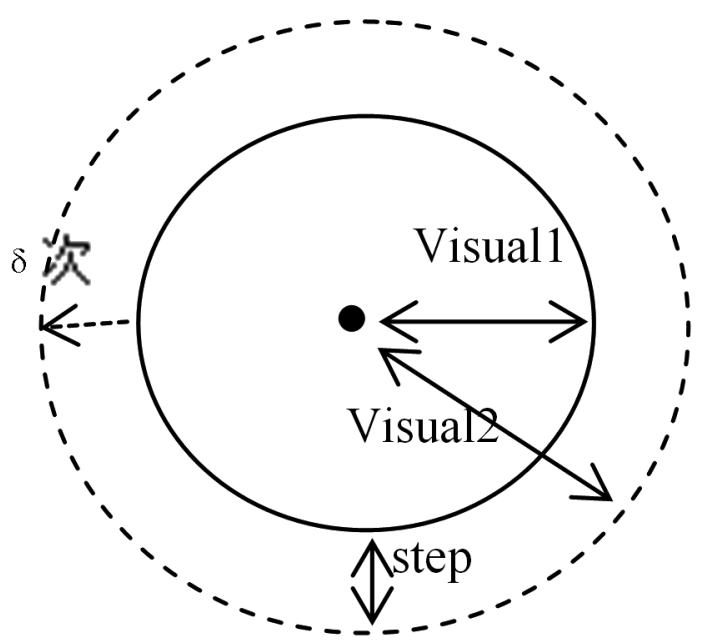

Fig. 1. Visual change Process

w. maxiter,curiter represent the maximum and current iterations number respectively.

\subsection{Variable neighborhood structure}

Artificial fish swarm algorithm(AFSA) is firstly proposed by Li Xiaolei in 2002[7]. In AFSA, each artificial fish has vision and can consider a step forward to the direction better than current position. The vision mechanism of artificial fish is firstly lead into particle swarm optimization after inspired by the optimization process in AFSA. In this paper, each particle is assigned a vision with a limited range, denoted by visual. The other particles within visual of a given particle form the neighborhood of it. In the standard PSO, every particle is affected by both individual and population experience in solution space. In order to promote the capabilities of information exchange and sharing, a new component is added into the velocity formula which represents the attraction generated from the neighborhood optimum. The new formula is as follow:

$$
\begin{gathered}
v_{i d}^{k+1}=w v_{i d}^{k}+c_{1} r_{1}\left(\alpha\left(\text { pbest }_{i d}-x_{i d}^{k}\right)+\right. \\
\left.(1-\alpha)\left(\text { nbest }_{i d}-x_{i d}^{k}\right)\right)+c_{2} r_{2}\left(\text { gbest }_{g d}-x_{i d}^{k}\right)
\end{gathered}
$$

Where $\alpha$ is a random number in the range $[0,1]$ and nbest $_{\text {id }}$ represents the best neighbourhood optimum found by particle $x_{i}$ : so far. The update formula of neighbourhood optimum with $x_{i}$ is as follow, for every particle $x_{i}$ : If

$$
\begin{aligned}
& \mid \begin{array}{l}
x_{j}-x_{i} \mid<\text { Visual } \\
\text { then }
\end{array} \\
& \qquad \text { nbest }_{i}=x_{j}
\end{aligned}
$$

In order to improve the local and global searching ability in standard PSO, In this pa- 
per, we propose the idea that all particles have gradient vision. The field change of particle vision is not happened in each iteration, but in certain quantity interations and was the ladder-like distribution. The advantage of this design is that particles can search optimal value in relative fixation neighborhood field and avoid missing a better search path due to frequently shift with vision. In the beginning iteration, the neighborhood of a particle is itself. When the iterative process is end, the neighborhood of a particle is all the swarm. The vision of a particle is defined as:

$$
\text { Visual }=\text { fix }(\text { curiter }, \delta) \times \text { step }
$$

Where curiter is the current iterative number, $\delta$ is interval times before next change of vision, fix is a reminder function, step represents each growth of the visual field. As shown in Fig.(1), the neighborhood field is solid circle when the vision of a particle is equal to Visual1. After iterations, the vision of the particle is update to Visual2 and the corresponding neighborhood filed is dashed circle. It satisfy that Visual2 $=$ Visual1 + step.

\subsection{Mutation operator}

If the optimal fitness value of the algorithm does not change through certain times of successive iterations, the algorithm is likely to converge to a local optimum of the problem, Paper [14] introduce reinitialization will occur aimed at arbitrary one-dimension of the current optimum. It is used to change the current searching trajectory so that particles can go out of the local optima and improve the accuracy of optimization.

This paper applies such effective and convenient operator to the improved PSO.. Let Rmax be a predefined constant that represents the allowed maximum iteration number. That is to say, if the optimal fitness value of the algorithm does not change through Rmax times of successive iterations, we choose a arbitrary dimension and replace it by a random number within $[-1,1]$ so as to enhance the exploration ability for particles and escape from the local optimum for the algorithm.

\section{Optimization for BP neural network}

\subsection{BP neural network}

BP neural network is a multilayer forward network for unidirectional propagation with three layers or more than three layers, Including the input layer, hidden layer and output layer and it is the most widely used neural network. In the use of BP neural network, the neural network must be given a set of learning samples in advance. Each neuron in the neu-

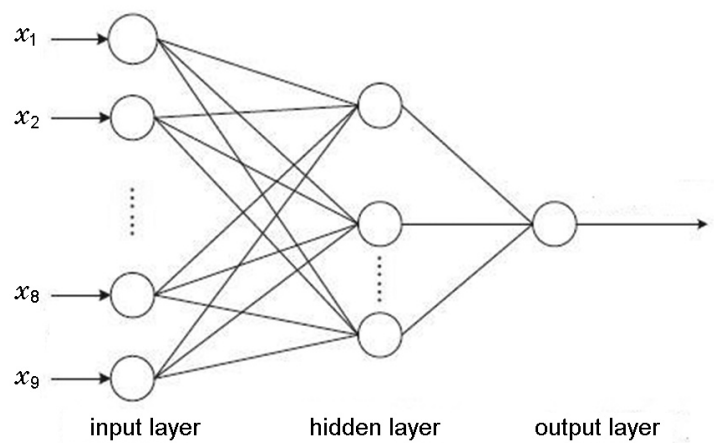

Fig. 2. Forcasting model of BP neural network

ral network contains a threshold and there is also weights between neurons. The input parameters are transferred from the input layer through the hidden layer to the output layer under the action of the corresponding weights and thresholds as well as the excitation function. BP neural network adjust the connection weights voluntarily through the training according to the minimum error criterion between the input and output data. Through continuously iteration, the nonlinear mapping between input and output is finally obtained.

But there are some disadvantages of $\mathrm{BP}$ neural network, such as it is easy to fall into local extreme value, slow convergence rate, randomicity of parameters selection, which can easily lead to the instability of the network. In view of the shortcomings of BP neural network, this paper use the improved particle optimization algorithm to determine the weights and threshods, and bring about prediction of silicon content in hot metal. Fig. 2 is a forcasting model of BP neural network.

\subsection{Encoding for weights and thresholds}

This paper use the variable neighborhood particle swarm optimization algorithm(VNPSO) to determine the weights and threshods of BP neural network. In the trainning process, all the weights and threshods in BP neural network are encoded as real number string and represented by a particle, etc. $x_{i}=\left(x_{i 1}, x_{i 2}, \cdots, x_{i L}\right)$, $i=1,2, \cdots, N, x_{i j}$ is initialized to the value between $[-1,1], L$ is defined as:

$$
L=\mathrm{In} \times \mathrm{Hi}+\mathrm{Hi} \times \mathrm{Ou}+\mathrm{Hi}+\mathrm{Ou}
$$

Where In, $\mathrm{Hi}$ and $\mathrm{Ou}$ are the number of input layer, hidden layer and output layer respectively.

In the iterations, each particle in the swarm is decomposed to weights and thresholds in neural network and brought into the neural network model to compute fitness of the particle repeatedly, until achieve the maximum number of iterations or obtain the permissible 
precision. The optimal particle is the training result of BP neural network.

The individual fitness fitness $s_{i}$ in swarm is defined as the mean square error of given sample:

$$
\text { fitness }_{i}=\frac{\sum_{j=1}^{M}\left(y_{j}-o_{j}\right)^{2}}{M}
$$

Where $M$ is the Number of samples for testing, are predictive and actual value corresponding to the ith sample respectively.

\section{Optimization steps}

The following steps are given to optimize the weights of BP neural network by the variable neighborhood particle swarm optimization algorithm:

Step 1: Initialize parameters: the population size $N$, the maximum iteration number LoopCount, the acceleration coefficients $c_{1}, c_{2}$, the interval frequency $\delta$, increment step, inertia weight w, Rmax, the constriction coefficient $\mathrm{X}$, the max velocity $v_{\max }$.

Step 2: Initialize position and velocity of all the particles randomly in the $D$ dimension space.

Step 3: Evaluate the fitness value of each particle, and update the global optimum position gbest, the neighborhood optimum position and the individual optimum position pbest .

Step 4: Update the inertia weight according to formula (3).

Step 5: Update the visual of population according to formula (6).

Step 6: For each particle, update particle velocity according formula (4), update particle position according formula (2).

1) If the current fitness value is better than pbest, assign the current position to pbest.

2) If the current best fitness value is better than the gbest, assign the current best position to gbest.

For each particle in a given one's neighbourhood, if the current fitness value of the particle is better than the nbest of the given one, assign the particle's current position to nbest corresponding to the given particle.

Step 7: Identify whether the algorithm has stagnated during successive Rmax times iterations. If the algorithm has stagnated, initialize any one-dimension of the optimal position by mutation operator.

Step 8: Repeat Step 4 - 8 until a stop criterion is satisfied or a predefined number of iterations are completed.

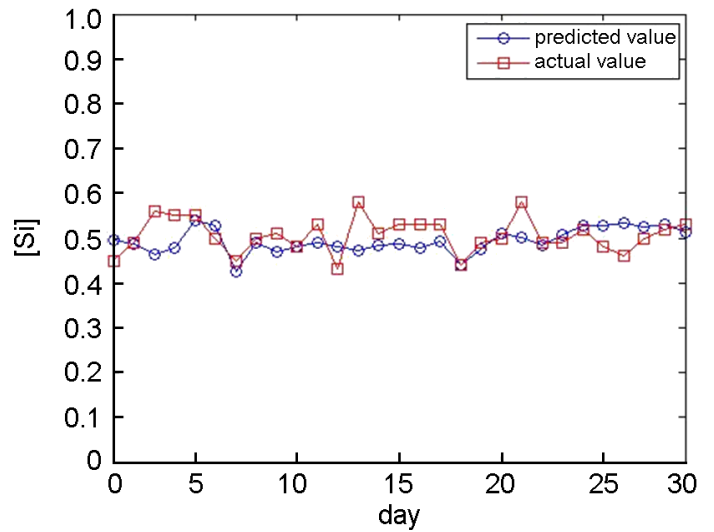

Fig. 3. Comparison between predicted and actual value

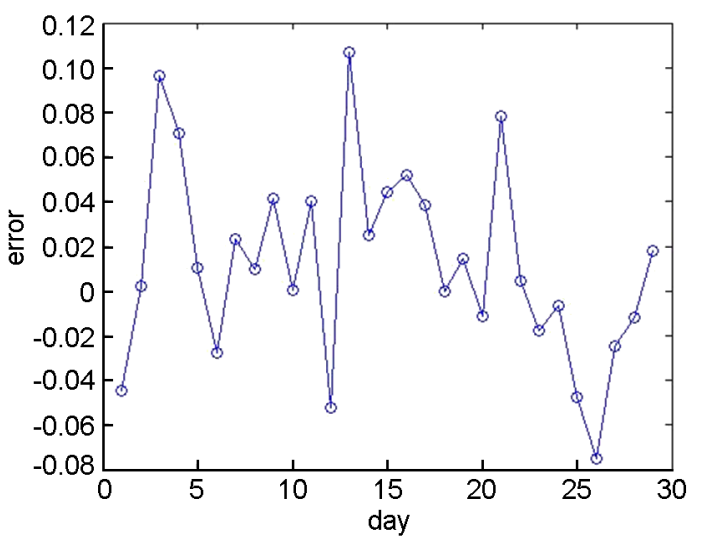

Fig. 4. Prediction error

\section{Prediction for Si content in hot metal}

This paper create a prediction model for si content in hot metal based on BP network optimized by Variable neighborhood PSO.Nine parameters that related to si content in hot metal were selected as input parameters to neural network from iron making in the blast furnace. They are air volume, hot blast drang, pressure difference, ventilating index, air temperature, top temperature, Oxygen enrichment, coal injection amount, batch nunber. The number of hidden layers is 10 and the output parameter is si content in hot metal. Number of particle swarm $N=60$. The dimension of particle swarm is 111 and each of them represents a weight or threshold. Due to the difference of these parameters, the input and output parameters are normalized in order to ensure that these factors are in the same position, the initial range of the weights is $[-1,1]$. The initial parameters is set as: $N=60, w_{\max }=0.9, w_{\min }=0.4 c_{1}=2$ ,$c_{2}=2$, loopcount $=200, \delta=10$, step $=0.15$.

Production data of every days that collected from a steel works in China in the first half of year 2012 is selected as training data set, 
containing 174 group data. A total of 29 group data in July is used as a test data set. The comparison between predicted and actual values is shown in Fig. 3. The model can predict the change trend of Si content in hot metal. The average relative error is $6.7 \%$., the accuracy was $97 \%$ when the errors was limited in the range of \pm 0.1 and the accuracy was $76 \%$ when the errors was limited in the range of \pm 0.05 . Prediction error is shown in Fig.(4).

\section{Conclusions}

The prediction accuracy of BP neural network is largely determined by its relative weights and thresholds. In this paper, the idea of variable neighborhood particle swarm optimization algorithm is proposed by introducing the vision mechanism. Then the improved algorithm is used to optimize the weights and thresholds of BP neural network so as to avoid falling into local extremum. Finally, this method is applied to the prediction of silicon content in hot metal. Through training and verification of the actual production data of a steel works, the average relative error of the prediction model is $6.7 \%$, the accuracy was $97 \%$ when the errors was limited in the range of $\pm \mathbf{0 . 1}$ and the accuracy was $76 \%$ when the errors was limited in the range of $\pm \mathbf{0 . 0 5}$.

\section{Acknowledgements}

This work was financially supported by scientific research fund of Liaoning provincial education department(L2014118) and The scientific research special fund of university of science and technology Liaoning(2015TD03).

\section{References}

1. Bi Xuegong, Mathematical model and computer control of blast furnace process[M], Beijing: China Metallurgical Industry Press, 1996:1.

2. Liu Xiangguan, Liu Fang. BF Ironmaking Process Optimization and Intelligent Control System[M]. Beijing: China Metallurgical Inustry Press, 2003.

3. Gao Xu-dong, China Metallurgy, 24, 24, 2014

4. Wang Hua-qiang, Gu Jin-chen, J. EFEI Univ. Technol., 31, 73, 2008

5. Yu Zhuoying, Zheng Tao, Hebei Metallurg. GY, 3, 38, 2015

6. Wang Wen-hui, Liu Xiang-guan, Metallurg. Indust.Autom. 38, 33, 2014

7. Li Xiao-lei,Shao zhi-jiang, Qian ji-xin Syst.Eng. Theor.Pract., 11,32,2002

8. Hai-mei Z, Yong-ping W., Control and Decision, 25, 20, 2010 . 